CARPATHIAN J. MATH.

Volume 37 (2021), No. 3,

Pages 407 - 416
Online version at https : //www . carpathian. cunbm . utcluj. ro/

Print Edition: ISSN 1584 - 2851; Online Edition: ISSN 1843 - 4401

DOI: https://doi.org/10.37193/CJM.2021.03.04

\title{
Measures of noncompactness and infinite systems of integral equations of Urysohn type in $L^{\infty}(\mathfrak{G})$
}

\author{
Shahram BANAEI ${ }^{1}$, VAhid PARVANEH ${ }^{2}$ and Mohammad MursaleEN ${ }^{3,4}$
}

\begin{abstract}
In this article, applying the concept of measure of noncompactness, some fixed point theorems in the Fréchet space $L^{\infty}(\mathfrak{G})$ (where $\mathfrak{G} \subseteq \mathbb{R}^{\omega}$ ) have been proved. We handle our obtained consequences to inquiry the existence of solutions for infinite systems of Urysohn type integral equations. Our results extend some famous related results in the literature. Finally, to indicate the effectiveness of our results we present a genuine example.
\end{abstract}

\section{INTRODUCTION AND PRELIMINARIES}

Measure of noncompactness (MNC) approaches ([8], [17]) have an substantial role in nonlinear functional analysis and fixed point theory. Heretofore, applying MNC approaches many articles have been extracted on the existence and behavior of solutions for nonlinear differential and integral equations. Some of these papers are $[2,3,6,7,11,14]$.

In this paper, we extract some fixed point theorems in Fréchet spaces with the assistance of MNC approaches and the Tychonoff fixed point theorem (TFPT), which are extensions of the results presented in [18, 19, 20,21].

The conformation of this paper is as follows. In part 1 , some preliminaries and concepts are summoned. Part 2 is allocated to stating some fixed point theorems of Darbo-type in the space $L^{\infty}(\mathfrak{G})$. Finally, in part 3, we apply our results to contemplate the existence of solutions for the following infinite system of nonlinear integral equations:

$$
\sigma_{n}(\iota)=\rho_{n}\left(\iota, \sigma_{1}(\iota) \ldots, \sigma_{n}(\iota), \ldots, \int_{\mathfrak{G}} \eta_{n}\left(\iota, \kappa,\left(\sigma_{j}(\kappa)\right)_{j=1}^{\infty}\right) d \kappa\right)
$$

where $\mathfrak{G} \subseteq \mathbb{R}^{\omega}$ in which $\mathbb{R}^{\omega}$ denotes the countable cartesian product of $\mathbb{R}$ with itself. Note that some classes of infinite system of nonlinear integral equations have been investigated in $[10,12,15]$.

All over this paper, $\mathfrak{B}$ is assumed to be an infinite dimensional Banach space or a Fréchet space. As well as, $\bar{B}(x, r)$ marks the closed ball centered at $x$ with radius $r$. The symbol $\bar{B}_{r}$ stands for the ball $\bar{B}(0, r)$. If $\mathcal{Q}$ be a subset of $\mathfrak{B}$, then the closure and closed convex hull of $\mathcal{Q}$, are announced by $\overline{\mathcal{Q}}$ and $\operatorname{Conv} \mathcal{Q}$, respectively. Furthermore, the family of all nonempty bounded subsets and the collection of all relatively compact subsets of $\mathfrak{B}$ are indicated by $\mathfrak{M}_{\mathfrak{B}}$ and $\mathfrak{N}_{\mathfrak{B}}$, respectively.

A vector space $\mathcal{Q}$ over the field $\mathbb{R}$ which is endowed with a topology such that the maps $(\iota, \kappa) \rightarrow \iota+\kappa$ and $(v, \iota) \rightarrow v \iota$ are continuous from $\mathcal{Q} \times \mathcal{Q}$ and $\mathbb{R} \times \mathcal{Q}$ to $\mathcal{Q}$ is called a topological vector space (TVS). A TVS is called locally convex if the origin has a neighborhood basis (i.e. a local base) consisting of convex sets [16]. Fréchet spaces are locally convex and complete with respect to a translation invariant metric.

Received: 19.11.2020. In revised form: 16.04.2021. Accepted: 23.04.2021

2010 Mathematics Subject Classification. 47H08, 47H10.

Key words and phrases. Measure of noncompactness, Tychonoff fixed point theorem, Fréchet space, System of integral equations.

Corresponding author: Sh. Banaei; math.sh.banaei@gmail.com. 
Example 1.1. [4] If $\mathfrak{B}_{i}$ is a Banach space $(i \in \mathbb{N})$ and $d: \prod_{i \in \mathbb{N}} \mathfrak{B}_{i} \times \prod_{i \in \mathbb{N}} \mathfrak{B}_{i} \longrightarrow \mathbb{R}$ be characterized by $d(\iota, \kappa)=\sup \left\{\frac{1}{2^{i}} \min \left\{1, d\left(\iota_{i}, \kappa_{i}\right)\right\}: i \in \mathbb{N}\right\}, \iota=\left(\iota_{1}, \iota_{2}, \ldots\right)$ and $\kappa=$ $\left(\kappa_{1}, \kappa_{2}, \ldots\right)$, then $\prod_{i \in \mathbb{N}} \mathfrak{B}_{i}$ is a Fréchet space.

Definition 1.1. [5] Let $\mathcal{M}$ be a collection of subsets of a Fréchet space $\mathfrak{B}$. If $\mathcal{Q} \in \mathcal{M}$ implies that $\operatorname{Conv}(\mathcal{Q}), \overline{\mathcal{Q}} \in \mathcal{M}$, then we say that $\mathcal{M}$ is an admissible set.

Definition 1.2. [5] Let $\mathcal{M}$ be an admissible subset of a Fréchet space $\mathfrak{B}$ and $\mathfrak{M}: \mathcal{M} \longrightarrow$ $\mathbb{R}_{+}$. We say that $\mathfrak{M}$ is a measure of noncompactness on $\mathfrak{B}$ if

$1^{\circ}$ The family $\operatorname{ker}\{\mathfrak{M}\}=\{\mathcal{Q} \in \mathcal{M}: \mathfrak{M}(\mathcal{Q})=0\}$ is nonempty and $\operatorname{ker}\{\mathfrak{M}\} \subseteq \mathfrak{N}_{\mathfrak{B}}$.

$2^{\circ} \mathcal{Q} \subset \Lambda \Longrightarrow \mathfrak{M}(\mathcal{Q}) \leq \mathfrak{M}(\Lambda)$.

$3^{\circ} \mathfrak{M}(\overline{\mathcal{Q}})=\mathfrak{M}(\mathcal{Q})$.

$4^{\circ} \mathfrak{M}(\operatorname{Conv} \mathcal{Q})=\mathfrak{M}(\mathcal{Q})$.

$5^{\circ} \mathfrak{M}(\eta X+(1-\eta) \Lambda) \leq \eta \mathfrak{M}(X)+(1-\eta) \mathfrak{M}(\Lambda)$ for all $\eta \in[0,1]$.

$6^{\circ}$ If $\left(\mathcal{Q}_{k}\right)$ be a sequence of closed sets from $\mathcal{M}$ such that $\mathcal{Q}_{k+1} \subset \mathcal{Q}_{k}$ for all $k=$ $1,2, \cdots$ and if $\lim _{k \rightarrow \infty} \mathfrak{M}\left(\mathcal{Q}_{k}\right)=0$, then $\mathcal{Q}_{\infty}=\cap_{k=1}^{\infty} \mathcal{Q}_{k} \neq \emptyset$.

Theorem 1.1. (TFPT [1]) Let $\mathfrak{B}$ be a Hausdorff locally convex $T V S, \mathcal{G}$ be a convex subset of $\mathfrak{B}$ and $H: \mathcal{G} \longrightarrow \mathfrak{B}$ be a continuous mapping such that

$$
H(\mathcal{G}) \subseteq A \subseteq \mathcal{G}
$$

where $A$ is compact. Then, $H$ possesses at least one fixed point.

\section{SOME FIXED POINT THEOREMS IN A FRÉCHET SPACE}

In this section, some Darbo-type fixed point theorems [9] in a Fréchet space have been investigated.

Theorem 2.2. Let $\mathcal{G}$ be a nonempty, closed and convex subset of a Fréchet space $\mathfrak{B}, \mathfrak{M}$ be a measure of noncompactness on $\mathfrak{B}$ and $H: \mathcal{G} \longrightarrow \mathcal{G}$ be a continuous mapping such that

$$
\mathfrak{M}(H(\mathcal{Q})) \leq \zeta(\mathfrak{M}(\mathcal{Q}))
$$

where $\mathcal{Q} \in \mathcal{M}$ and $\zeta: \mathbb{R}_{+} \longrightarrow \mathbb{R}_{+}$is a nondecreasing function such that $\lim _{n \rightarrow \infty} \zeta^{n}(t)=0$ for all $t>0$. Then $H$ admits at least a fixed point in $\mathcal{G}$.

Note that $\zeta(t)=k t$ for some $k \in(0,1)$ and $\zeta(t)=\ln (1+t / 2)$ are some examples of function $\zeta$.

Proof. Construct $\left(\mathcal{P}_{n}\right)$ such that $\mathcal{P}_{0}=\mathcal{G}$ and $\mathcal{P}_{n}=\operatorname{Conv} H\left(\mathcal{P}_{n-1}\right)$ for all $n \geq 1$, inductively. According to the suppositions of the mapping $\mathfrak{M}$, we have

$$
\mathfrak{M}\left(\mathcal{P}_{n}\right)=\mathfrak{M}\left(\operatorname{Conv} H\left(\mathcal{P}_{n-1}\right)\right)=\mathfrak{M}\left(H\left(\mathcal{P}_{n-1}\right)\right) \leq \zeta\left(\mathfrak{M}\left(\mathcal{P}_{n-1}\right)\right) .
$$

Therefore,

$$
\mathfrak{M}\left(\mathcal{P}_{n}\right) \leq \zeta^{n}\left(\mathfrak{M}\left(\mathcal{P}_{0}\right)\right)
$$

Abandoning $n \longrightarrow \infty$ in the above relation, we understand that $\lim _{n} \longrightarrow \infty \mathfrak{M}\left(\mathcal{P}_{n}\right)=0$. If $\mathcal{P}_{\infty}=\cap_{n=1}^{\infty} \mathcal{P}_{n}$, then Definition 1.2 warrantees that $\mathcal{P}_{\infty}$ is nonempty. Obviously, $\mathcal{P}_{\infty}$ is a convex compact subset of $L^{\infty}(\mathfrak{G})$. Now, TFPT insinuates that $H$ possesses a fixed point. 
Corollary 2.1. Let $\mathcal{G}$ be a nonempty, closed and convex subset of a Fréchet space $\mathfrak{B}$, $\mathfrak{M}$ be a measure of noncompactness on $\mathfrak{B}$ and $H: \mathcal{G} \longrightarrow \mathcal{G}$ be a continuous mapping such that

$$
\mathfrak{M}(H(\mathcal{Q})) \leq k \mathfrak{M}(\mathcal{Q}),
$$

where $\mathcal{Q} \in \mathcal{M}$ and $k \in[0,1)$. Then $H$ admits at least a fixed point in $\mathcal{G}$.

Theorem 2.3. Suppose that $\mathfrak{M}_{i}$ be a measure of noncompactness on Banach space $\mathfrak{B}_{i}$ for all $i \in \mathbb{N}$. Let

$$
\mathcal{M}=\left\{\mathcal{G} \subseteq \prod_{i=1}^{\infty} \mathfrak{B}_{i}: \sup _{i \geq 1}\left\{\mathfrak{M}_{i}\left(\varpi_{i}(\mathcal{G})\right)\right\}<\infty\right\}
$$

where $\varpi_{i}(\mathcal{G})$ announces the natural projection of $\prod_{i=1}^{\infty} \mathfrak{B}_{i}$ into $\mathfrak{B}_{i}$ and $\widetilde{\mathfrak{M}}: \mathcal{M} \longrightarrow \mathbb{R}_{+}$be defined by

$$
\widetilde{\mathfrak{M}}(\mathcal{G})=\sup _{i \geq 1}\left\{\mathfrak{M}_{i}\left(\varpi_{i}(\mathcal{G})\right)\right\}
$$

then $\widetilde{\mathfrak{M}}$ is a measure of noncompactness on $\mathcal{Q}=\prod_{i=1}^{\infty} \mathfrak{B}_{i}$.

Proof. The proof of $\left(2^{\circ}\right)$ is obvious and the properties $\left(3^{\circ}\right)-\left(5^{\circ}\right)$ are immediate consequences of

$$
\begin{aligned}
& \varpi_{i}(\alpha \mathcal{U}+(1-\alpha) \mathcal{V})=\alpha \varpi_{i}(\mathcal{U})+(1-\alpha) \varpi_{i}(\mathcal{V}), \\
& \varpi_{i}(\operatorname{Conv} \mathcal{G})=\operatorname{Conv} \varpi_{i}(\mathcal{G}), \\
& \varpi_{i}(\mathcal{G}) \subseteq \varpi_{i}(\overline{\mathcal{G}}) \subseteq \overline{\varpi_{i}(\mathcal{G})}
\end{aligned}
$$

If $\widetilde{\mathfrak{M}}(\mathcal{G})=0$ for some $\mathcal{G} \in \mathcal{M}$, then $\mathfrak{M}_{i}\left(\varpi_{i}(\mathcal{G})\right)=0$ for each $1 \leq i \leq n$. Hence, according to $\left(1^{\circ}\right)$ of Definition 1.2 for the measure of noncompactness $\mathfrak{M}_{i}$ we deduce that $\varpi_{i}(\mathcal{G})$ is relatively compact for all $i \in \mathbb{N}$. Now, exploiting the Tychonoff's theorem[13], we see that $\mathcal{G}$ is relatively compact. Eventually, it sufficient to show $\left(6^{\circ}\right)$. Let $\left(\mathcal{G}_{n}\right)$ be a sequence of closed sets from $\mathcal{M}$ such that $\mathcal{G}_{n+1} \subseteq \mathcal{G}_{n}$ for all $n \in \mathbb{N}$ and $\lim _{n \rightarrow \infty} \widetilde{\mathfrak{M}}\left(\mathcal{G}_{n}\right)=0$ for all $n \in \mathbb{N}$. So, we conclude that $\lim _{n \rightarrow \infty} \mathfrak{M}_{i}\left(\varpi_{i}\left(\mathcal{G}_{n}\right)\right)=0$ (or, $\lim _{n \longrightarrow \infty} \mathfrak{M}_{i}\left(\varpi_{i}\left(\mathcal{G}_{n}\right)\right)=0$ ) and $\mathcal{Q}_{i}^{\infty}=\bigcap_{n=1}^{\infty} \varpi_{i}\left(\mathcal{G}_{n}\right) \neq \emptyset$ for all $i \in \mathbb{N}$. Therefore, $\prod_{i=1}^{\infty} \mathcal{Q}_{i}^{\infty}=\mathcal{G}_{\infty} \neq \emptyset$. This ends the proof.

Remark 2.1. The Proof of Theorems 2.2 and 2.3 are parallel to the Proof of Theorems 3.2 and 3.1 of reference [5], respectively.

Corollary 2.2. Let $B_{i}(i \in \mathbb{N})$ be a nonempty, closed, convex and bounded subset of Banach space $\mathfrak{B}_{i}$ and $\mathfrak{M}_{i}$ be an arbitrary $(M N C)$ on $\mathfrak{B}_{i}$. Let $H_{i}: \prod_{i=1}^{\infty} B_{i} \longrightarrow B_{i}(i \in \mathbb{N})$ be a continuous operator such that

$$
\mathfrak{M}_{i}\left(H_{i}\left(\prod_{i=1}^{\infty} \mathcal{Q}_{i}\right)\right) \leq\left(\sup _{i \geq 1} \zeta_{i}\right)\left(\mathfrak{M}_{i}\left(\mathcal{Q}_{i}\right)\right),
$$

where $\mathcal{Q}_{i} \subseteq B_{i}(i \in \mathbb{N})$ and $\zeta_{i}: \mathbb{R}_{+} \longrightarrow \mathbb{R}_{+}$is a bounded mapping which satisfies the conditions of Theorem 2.2 for all $i \in \mathbb{N}$. Then there exists $\left(\iota_{j}^{*}\right)_{j=1}^{\infty} \in \prod_{j=1}^{\infty} B_{j}$ such that

$$
H_{i}\left(\left(\iota_{j}^{*}\right)_{j=1}^{\infty}\right)=\iota_{i}^{*},
$$

for all $i \in \mathbb{N}$. 
Proof. Let $\widetilde{H}: \prod_{i=1}^{\infty} B_{i} \longrightarrow \prod_{i=1}^{\infty} B_{i}$ be characterized by

$$
\widetilde{H}\left(\left(\iota_{j}\right)_{j=1}^{\infty}\right)=\left(H_{1}\left(\left(\iota_{j}\right)_{j=1}^{\infty}\right), H_{2}\left(\left(\iota_{j}\right)_{j=1}^{\infty}\right), \ldots, H_{i}\left(\left(\iota_{j}\right)_{j=1}^{\infty}\right), \ldots\right)
$$

for all $\left(\iota_{j}\right)_{j=1}^{\infty} \in \prod_{i=1}^{\infty} B_{i}$. We will investigate that all conditions of Theorem 2.2 hold. Let $\mathcal{Q} \subseteq \prod_{i=1}^{\infty} B_{i}$. Let $\varpi_{i}(\mathcal{Q})$ signifies the natural projection of $\prod_{i=1}^{\infty} \mathcal{Q}_{i}$ into $\mathcal{Q}_{i}$. For each $n \in \mathbb{N}$, we have

$$
\begin{aligned}
\widetilde{\mathfrak{M}}(\widetilde{H}(\mathcal{Q})) & \leq \widetilde{\mathfrak{M}}\left(\widetilde{H}\left(\prod_{k=1}^{\infty} \varpi_{k}(\mathcal{Q})\right)\right) \\
& =\sup _{i \geq 1}\left\{\mathfrak{M}_{i}\left(H_{i}\left(\prod_{k=1}^{\infty} \varpi_{k}(\mathcal{Q})\right)\right)\right\} \\
& \left.\leq \sup _{i \geq 1} \zeta_{i}\right)(\widetilde{\mathfrak{M}}(\mathcal{Q})) .
\end{aligned}
$$

Taking $\widetilde{\zeta}=\sup _{i>1} \zeta_{i}$, all conditions of Theorem 2.2 are satisfied. Therefore, $\widetilde{H}$ possesses a fixed point and (2.6) holds.

\section{Application}

In this section, we study the existence of a solution for integral equation system (1.1) in the space $L^{\infty}(\mathfrak{G})$, where $\mathfrak{G} \subseteq \mathbb{R}^{\omega}$ to indicate the applicability of presented results.

Let $L^{\infty}(\mathfrak{G})$ be the space of all real valued Lebesgue measurable functions on an open subset $\mathfrak{G}$ of $\mathbb{R}^{\omega}$ which are essentially bounded on $\mathbb{R}^{\omega}$, endowed with the norm

$$
\|\rho\|_{\infty}=\inf \{M>0:|\rho| \leq M \text { a.e. on } \mathfrak{G}\} .
$$

Let $\mathcal{Q}$ be a bounded subset of the space $L^{\infty}(\mathfrak{G})$. Let $\mathcal{Z}$ be a positive real number and $\bar{B}_{\mathcal{Z}}$ be the closed ball with center 0 and radius $\mathcal{Z}$. For all $\rho \in \mathcal{Q}$ and for all $\varepsilon>0$, let:

$$
\begin{aligned}
& \omega^{\mathcal{Z}}(\rho, \varepsilon)=\sup \left\{\left\|v_{h} \rho-\rho\right\|_{L^{\infty}\left(\bar{B}_{\mathcal{Z}}\right)}:\|h\|<\varepsilon\right\} \\
& \omega^{\mathcal{Z}}(\mathcal{Q}, \varepsilon)=\sup \left\{\omega^{\mathcal{Z}}(\rho, \varepsilon): \rho \in \mathcal{Q}\right\}, \\
& \omega_{0}^{\mathcal{Z}}(\mathcal{Q})=\lim _{\varepsilon \longrightarrow 0} \omega^{\mathcal{Z}}(\mathcal{Q}, \varepsilon), \\
& \omega_{0}(\mathcal{Q})=\lim _{\mathcal{Z} \longrightarrow \infty} \omega_{0}^{\mathcal{Z}}(\mathcal{Q}),
\end{aligned}
$$

and

$$
\begin{aligned}
& d_{\mathcal{Z}}(\mathcal{Q})=\sup \left\{\text { ess } \sup _{\|\iota\|>\mathcal{Z}}|\rho(\iota)-\varrho(\iota)|: \rho, \varrho \in \mathcal{Q}\right\}, \\
& d(\mathcal{Q})=\lim _{\mathcal{Z} \longrightarrow \infty} d_{\mathcal{Z}}(\mathcal{Q}) \\
& \mathfrak{M}(\mathcal{Q})=\omega_{0}(\mathcal{Q})+d(\mathcal{Q}) .
\end{aligned}
$$

The function $\mathfrak{M}$ is a measure of noncompactness in the space $L^{\infty}(\mathfrak{G})$ [3].

Definition 3.3. A function $\rho: \mathfrak{G} \times \mathbb{R}^{\omega} \longrightarrow \mathbb{R}$ is said to have the Carathéodory property if

(a) The function $\iota \longrightarrow \rho(\iota, \sigma)$ is measurable on $\mathfrak{G}$ for all $\sigma \in \mathbb{R}^{\omega}$.

(b) The function $\sigma \longrightarrow \rho(\iota, \sigma)$ is continuous on $\mathbb{R}^{\omega}$ for almost all $\iota \in \mathbb{R}^{\omega}$.

Let:

$\left(\mathcal{P}_{1}\right) \rho_{n}: \mathfrak{G} \times \mathbb{R}^{\omega} \times \mathbb{R} \longrightarrow \mathbb{R}\left(n \in \mathbb{N}\right.$ and $\mathfrak{G}$ is an unbounded subset of $\left.\mathbb{R}^{\omega}\right)$ satisfies the Carathéodory conditions with $\rho_{n}(., 0, \ldots) \in L^{\infty}(\mathfrak{G})$. Moreover, for a bounded nondecreasing, concave and upper semicontinuous mapping $\chi$ with $\chi(t)<t$,

$$
\left|\rho_{n}\left(\iota, \sigma_{1}, \ldots, \sigma_{n}, \ldots, \varrho\right)-\rho_{n}\left(\iota, \varsigma_{1}, \ldots, \varsigma_{n}, \ldots, q\right)\right| \leq \chi\left(\sup _{i \geq 1}\left|\sigma_{i}-\varsigma_{i}\right|\right)+|\varrho-q|,
$$

for a.e. $\iota \in \mathfrak{G}$. 
$\left(\mathcal{P}_{2}\right) \eta_{n}: \mathfrak{G} \times \mathfrak{G} \times \mathbb{R}^{\omega} \longrightarrow \mathbb{R}(n \in \mathbb{N})$ satisfies the Carathéodory conditions, $\eta_{n} \in$ $L_{\text {loc }}^{\infty}\left(\mathfrak{G} \times \mathfrak{G} \times \mathbb{R}^{\omega}\right)$ and for a positive constant $D$

$$
\text { ess } \sup _{\iota \in \mathfrak{G}}\left\{\left|\int_{\mathfrak{G}} \eta_{n}\left(\iota, \kappa,\left(\sigma_{j}(\kappa)\right)_{j=1}^{\infty}\right) d \kappa\right|\right\} \leq D .
$$

Moreover,

$$
\lim _{\mathcal{Z} \longrightarrow \infty} \text { ess } \sup _{\|\iota\|>\mathcal{Z}}\left|\int_{\mathfrak{G}}\left(\eta_{n}\left(\iota, \kappa,\left(\sigma_{j}(\kappa)\right)_{j=1}^{\infty}\right)-\eta_{n}\left(\iota, \kappa,\left(\varsigma_{j}(\kappa)\right)_{j=1}^{\infty}\right)\right) d \kappa\right|=0
$$

uniformly with respect to $\sigma_{j}, \varsigma_{j} \in L^{\infty}(\mathfrak{G})$ and for all $r>0$ with $\max \left\{\left\|\sigma_{j}\right\|_{\infty},\left\|\varsigma_{j}\right\|_{\infty}\right\} \leq r ;$

$$
\lim _{\mathcal{Z} \longrightarrow \infty} e s s \sup _{\iota \in \mathfrak{G}} \int_{\mathfrak{G} \backslash \bar{B}_{\mathcal{Z}}}\left|\eta_{n}\left(\iota, \kappa,\left(\sigma_{j}(\kappa)\right)_{j=1}^{\infty}\right)\right| d \kappa=0 .
$$

$\left(\mathcal{P}_{4}\right)$ For each $n \in \mathbb{N}$

$$
\chi\left(\sup _{i \geq 1} \lambda_{i}\right)+E_{n}+D \leq \lambda_{n_{0}}
$$

for some $n_{0}$ such that $\lambda_{n} \leq \lambda_{n+1}$ where $E_{n}:=$ ess $\sup _{\iota \in \mathfrak{G}}\left|\rho_{n}(\iota, 0,0, \ldots)\right|$.

Note that we say that a function $f: \mathcal{G} \longrightarrow \mathbb{R}$ belongs to $L_{\text {loc }}^{\infty}\left(\mathbb{R}^{\omega}\right)$ if $f \chi K \in L^{\infty}\left(\mathbb{R}^{\omega}\right)$ for every compact set $K$ contained in $\mathcal{G}$, where $\mathcal{G}$ is an open subset of $\mathbb{R}^{\omega}$.

Theorem 3.4. Having suppositions $\left(\mathcal{P}_{1}\right)-\left(\mathcal{P}_{4}\right)$, the infinite system (1.1) admits at least one solution $\left(\sigma_{i}=\sigma_{i}(\iota)\right)_{i=1}^{\infty} \in\left(L^{\infty}(\mathfrak{G})\right)^{\omega}$.

Proof. First, consider arbitrary $n \in \mathbb{N}$. Let $H_{n}:\left(L^{\infty}(\mathfrak{G})\right)^{\omega} \longrightarrow L^{\infty}(\mathfrak{G})$ be defined by

$$
H_{n}\left(\left(\sigma_{j}\right)_{j=1}^{\infty}\right)(\iota)=\rho_{n}\left(\iota, \sigma_{1}(\iota), \ldots, \sigma_{n}(\iota), \ldots, \int_{\mathfrak{G}} \eta_{n}\left(\iota, \kappa,\left(\sigma_{j}(\kappa)\right)_{j=1}^{\infty}\right) d \kappa\right) .
$$

According to the Carathéodory conditions, we conclude that $H_{n}\left(\left(\sigma_{j}\right)_{j=1}^{\infty}\right)$ is measurable for any $\left(\sigma_{j}\right)_{j=1}^{\infty} \in\left(L^{\infty}(\mathfrak{G})\right)^{\omega}$.

Now, we prove that $H_{n}\left(\left(\sigma_{j}\right)_{j=1}^{\infty}\right) \in L^{\infty}(\mathfrak{G})$. Handling suppositions $\left(\mathcal{P}_{1}\right)-\left(\mathcal{P}_{4}\right)$ we have

$$
\begin{aligned}
& \left|H_{n}\left(\left(\sigma_{j}\right)_{j=1}^{\infty}\right)(\iota)\right| \\
& \leq\left|\rho_{n}\left(\iota, \sigma_{1}(\iota), \ldots, \sigma_{n}(\iota), \ldots, \int_{\mathfrak{G}} \eta_{n}\left(\iota, \kappa,\left(\sigma_{j}(\kappa)\right)_{j=1}^{\infty}\right) d \kappa\right)-\rho_{n}(\iota, 0, \ldots)\right|+\left|\rho_{n}(\iota, 0, \ldots)\right| \\
& \leq \chi\left(\sup _{i \geq 1}\left|\sigma_{i}(\iota)\right|\right)+\left|\int_{\mathfrak{G}} \eta_{n}\left(\iota, \kappa,\left(\sigma_{j}(\kappa)\right)_{j=1}^{\infty}\right) d \kappa\right|+\left|\rho_{n}(\iota, 0, \ldots)\right| \\
& \leq \chi\left(\sup _{i \geq 1}\left\|\sigma_{i}\right\|_{\infty}\right)+D+E_{n} .
\end{aligned}
$$

for a.e. $\iota \in \mathfrak{G}$. Therefore, we conclude that

$$
\left\|H_{n}\left(\left(\sigma_{j}\right)_{j=1}^{\infty}\right)\right\|_{\infty} \leq \chi\left(\sup _{i \geq 1}\left\|\sigma_{i}\right\|_{\infty}\right)+D+E_{n} .
$$

Thus, $H_{n}\left(\left(\sigma_{j}\right)_{j=1}^{\infty}\right) \in L^{\infty}(\mathfrak{G})$ for any $\left(\sigma_{j}(\iota)\right)_{i=1}^{\infty} \in\left(L^{\infty}(\mathfrak{G})\right)^{\omega}$.

Propounding relation (3.12) and exploiting $\left(\mathcal{P}_{4}\right)$, the function $H_{n}$ maps $\prod_{i=1}^{\infty} \bar{B}_{\lambda_{i}}$ into $\bar{B}_{\lambda_{n_{0}}}$.

Now, we prove that $H_{n}$ is a continuous operator. Let $0<\varepsilon$ for which $0<\varepsilon<\frac{1}{2^{n}}$ and take arbitrary $\left(\left(\sigma_{j}\right)_{j=1}^{\infty}\right)$ and $\left(\left(\varsigma_{j}\right)_{j=1}^{\infty}\right) \in\left(L^{\infty}(\mathfrak{G})\right)^{\omega}$ such that

$$
d\left(\left(\left(\sigma_{j}\right)_{j=1}^{\infty}\right),\left(\left(\varsigma_{j}\right)_{j=1}^{\infty}\right)\right)=\sup \left\{\frac{1}{2^{i}} \min \left\{1,\left\|\sigma_{i}-\varsigma_{i}\right\|_{\infty}\right\}: i \in \mathbb{N}\right\}<\varepsilon .
$$

Then, 


$$
\begin{aligned}
& \mid H_{n}\left(\left(\sigma_{j}\right)_{j=1}^{\infty}\right)(\iota)- H_{n}\left(\left(\varsigma_{j}\right)_{j=1}^{\infty}\right)(\iota)|\leq| \rho_{n}\left(\iota, \sigma_{1}(\iota), \ldots, \sigma_{n}(\iota), \ldots, \int_{\mathfrak{G}} \eta_{n}\left(\iota, \kappa,\left(\sigma_{j}(\kappa)\right)_{j=1}^{\infty}\right) d \kappa\right) \\
&-\rho_{n}\left(\iota, \varsigma_{1}(\iota), \ldots, \varsigma_{n}(\iota), \ldots, \int_{\mathfrak{G}} \eta_{n}\left(\iota, \kappa,\left(\varsigma_{j}(\kappa)\right)_{j=1}^{\infty}\right) d \kappa\right) \mid \\
&\left.\leq \chi \sup _{i \geq 1}\left|\sigma_{i}(\iota)-\varsigma_{i}(\iota)\right|\right)+\mid \int_{\mathfrak{G}} \eta_{n}\left(\iota, \kappa,\left(\sigma_{j}(\kappa)\right)_{j=1}^{\infty} d \kappa-\int_{\mathfrak{G}} \eta_{n}\left(\iota, \kappa,\left(\varsigma_{j}(\kappa)\right)_{j=1}^{\infty}\right) d \kappa \mid .\right.
\end{aligned}
$$

Exerting supposition $\left(\mathcal{P}_{1}\right)$ we can choose $\mathcal{Z}_{1}>0$ such that

$$
\underset{\|\iota\|>\mathcal{Z}}{\operatorname{ess} \sup _{\mathfrak{G}}}\left|\int_{\mathfrak{G}}\left[\eta_{n}\left(\iota, \kappa,\left(\sigma_{j}(\kappa)\right)_{j=1}^{\infty}\right)-\eta_{n}\left(\iota, \kappa,\left(\varsigma_{j}(\kappa)\right)_{j=1}^{\infty}\right)\right] d \kappa\right|<\varepsilon .
$$

Exerting suppositions $\left(\mathcal{P}_{2}\right)$ we can choose $\mathcal{Z}_{2}>0$ such that

$$
\text { ess } \sup _{\iota \in \mathfrak{G}} \int_{\mathfrak{G} \backslash \bar{B}_{\mathcal{Z}}}\left|\eta_{n}\left(\iota, \kappa,\left(\sigma_{j}(\kappa)\right)_{j=1}^{\infty}\right)\right| d \kappa<\varepsilon .
$$

Now, let $\mathcal{Z}=\max \left\{\mathcal{Z}_{1}, \mathcal{Z}_{2}\right\}$.

From (3.13), we conclude that

$$
\underset{\|\iota\|>\mathcal{Z}}{\operatorname{ess} \sup }\left|H_{n}\left(\left(\sigma_{j}\right)_{j=1}^{\infty}\right)(\iota)-H_{n}\left(\left(\varsigma_{j}\right)_{j=1}^{\infty}\right)(\iota)\right| \leq \chi(\varepsilon)+\varepsilon .
$$

For almost all $\iota \in \bar{B}_{\mathcal{Z}} \cap \mathfrak{G}$, we have

$$
\begin{aligned}
& \left|H_{n}\left(\left(\sigma_{j}\right)_{j=1}^{\infty}\right)(\iota)-H_{n}\left(\left(\varsigma_{j}\right)_{j=1}^{\infty}\right)(\iota)\right| \leq \chi\left(\sup _{i \geq 1}\left|\sigma_{i}(\iota)-\varsigma_{i}(\iota)\right|\right) \\
& \quad+\left|\int_{\bar{B}_{\mathcal{Z}}}\left[\eta_{n}\left(\iota, \kappa,\left(\sigma_{j}(\kappa)\right)_{j=1}^{\infty}\right)-\eta_{n}\left(\iota, \kappa,\left(\varsigma_{j}(\kappa)\right)_{j=1}^{\infty}\right)\right] d \kappa\right| \\
& \quad+\left|\int_{\mathfrak{G} \backslash \bar{B}_{\mathcal{Z}}}\left[\eta_{n}\left(\iota, \kappa,\left(\sigma_{j}(\kappa)\right)_{j=1}^{\infty}\right)-\eta_{n}\left(\iota, \kappa,\left(\varsigma_{j}(\kappa)\right)_{j=1}^{\infty}\right)\right] d \kappa\right| \\
& \leq \chi(\varepsilon)+\left|\int_{\bar{B}_{\mathcal{Z}}}\left[\eta_{n}\left(\iota, \kappa,\left(\sigma_{j}(\kappa)\right)_{j=1}^{\infty}\right)-\eta_{n}\left(\iota, \kappa,\left(\varsigma_{j}(\kappa)\right)_{j=1}^{\infty}\right)\right] d \kappa\right| \\
& \quad+2 e s s \sup \int_{\mathfrak{G} \backslash \bar{B}_{\mathcal{Z}}}\left|\eta_{n}\left(\iota, \kappa,\left(\sigma_{j}(\kappa)\right)_{j=1}^{\infty}\right)\right| d \kappa \\
& \leq \chi(\varepsilon)+\vartheta_{n}(\varepsilon)+2 e s s \sup _{\iota \in \mathfrak{G}} \int_{\mathfrak{G} \backslash \bar{B}_{\mathcal{Z}}}\left|\eta_{n}\left(\iota, \kappa,\left(\sigma_{j}(\kappa)\right)_{j=1}^{\infty}\right)\right| d \kappa
\end{aligned}
$$

where

$$
\begin{aligned}
& \vartheta_{n}(\varepsilon)=\inf \left\{\mathcal{G} \geq 0:\left|\eta_{n}\left(\iota, \kappa,\left(\sigma_{j}(\kappa)\right)_{j=1}^{\infty}\right)-\eta_{n}\left(\iota, \kappa,\left(\varsigma_{j}(\kappa)\right)_{j=1}^{\infty}\right)\right| \leq \mathcal{G}\right. \\
& \text { for a.e. } \left.\iota, \kappa \in \bar{B}_{\mathcal{Z}} \subset \mathfrak{G}, \text { and }\left(\varsigma_{j}(\kappa)_{j=1}^{\infty}\right) \in \bar{B}\left(\left(\sigma_{j}(\kappa)_{j=1}^{\infty}\right), \varepsilon\right)\right\} .
\end{aligned}
$$

Exerting the Carathéodory reservations for $\eta_{n}$ on $\bar{B}_{\mathcal{Z}} \times \bar{B}_{\mathcal{Z}} \times \bar{B}\left(\left(\sigma_{j}(\kappa)_{j=1}^{\infty}\right), \varepsilon\right)$, one has $\vartheta_{n}(\varepsilon) \rightarrow 0$ as $\varepsilon \rightarrow 0$. Therefore, from (3.14), (3.15) and (3.16) we conclude that $H_{n}$ is continuous on $\left(L^{\infty}(\mathfrak{G})\right)^{\omega}$.

Now, we demonstrate that $H_{n}$ has all the reservations of Corollary 2.2. Let $\Delta_{n}$ be a nonempty subset of $\bar{B}_{\lambda_{n}}$ for all $n \in \mathbb{N}$ with $\Delta_{n} \subset \Delta_{n+1}$. Assume that $\mathcal{Z}>0$ and $\varepsilon>0$ be 
given. For almost all $\iota \in \mathfrak{G}$, all $h$ with $\|h\| \leq \varepsilon$ and all $\sigma_{n} \in \Delta_{n}$ we have

$$
\begin{aligned}
& \left|H_{n}\left(\left(\sigma_{j}\right)_{j=1}^{\infty}\right)(\iota)-H_{n}\left(\left(\sigma_{j}\right)_{j=1}^{\infty}\right)(\iota+h)\right| \\
& \leq \mid \rho_{n}\left(\iota, \sigma_{1}(\iota), \ldots, \sigma_{n}(\iota), \ldots \int_{\mathfrak{G}} \eta_{n}\left(\iota, \kappa,\left(\sigma_{j}(\kappa)\right)_{j=1}^{\infty}\right) d \kappa\right) \\
& -\rho_{n}\left(\iota+h, \sigma_{1}(\iota), \ldots, \sigma_{n}(\iota), \ldots \int_{\mathfrak{G}} \eta_{n}\left(\iota, \kappa,\left(\sigma_{j}(\kappa)\right)_{j=1}^{\infty}\right) d \kappa\right) \mid \\
& +\mid \rho_{n}\left(\iota+h, \sigma_{1}(\iota), \ldots, \sigma_{n}(\iota), \ldots \int_{\mathfrak{G}} \eta_{n}\left(\iota, \kappa,\left(\sigma_{j}(\kappa)\right)_{j=1}^{\infty}\right) d \kappa\right) \\
& -\rho_{n}\left(\iota+h, \sigma_{1}(\iota+h), \ldots, \sigma_{n}(\iota+h), \ldots, \int_{\mathfrak{G}} \eta_{n}\left(\iota+h, \kappa,\left(\sigma_{j}(\kappa)\right)_{j=1}^{\infty}\right) d \kappa\right) \mid \\
& \leq H_{\left\{\lambda_{n}\right\}}^{\mathcal{Z}}\left(\rho_{n}, \varepsilon\right)+\chi\left(\sup _{i \geq 1}\left|\sigma_{i}(\iota)-\sigma_{i}(\iota+h)\right|\right) \\
& +\left|\int_{\bar{B}_{\mathcal{Z}}} \eta_{n}\left(\iota, \kappa,\left(\sigma_{j}(\kappa)\right)_{j=1}^{\infty}\right)-\eta_{n}\left(\iota+h, \kappa,\left(\sigma_{j}(\kappa)\right)_{j=1}^{\infty}\right) d \kappa\right| \\
& +\left|\int_{\mathfrak{G} \backslash \bar{B}_{\mathcal{Z}}} \eta_{n}\left(\iota, \kappa,\left(\sigma_{j}(\kappa)\right)_{j=1}^{\infty}\right)-\eta_{n}\left(\iota+h, \kappa,\left(\sigma_{j}(\kappa)\right)_{j=1}^{\infty}\right) d \kappa\right| \\
& \leq H_{\left\{\lambda_{n}\right\}}^{\mathcal{Z}}\left(\rho_{n}, \varepsilon\right)+\chi\left(\sup _{i \geq 1} \omega^{\mathcal{Z}}\left(\sigma_{i}, \varepsilon\right)\right) \\
& +\omega_{\left\{\lambda_{n}\right\}}^{\mathcal{Z}}\left(\eta_{n}, \varepsilon\right)+2 e s s \sup _{\iota \in \mathfrak{G}} \int_{\mathfrak{G} \backslash \bar{B}_{\mathcal{Z}}}\left|\eta_{n}\left(\iota, \kappa,\left(\sigma_{j}(\kappa)\right)_{j=1}^{\infty}\right)\right| d \kappa,
\end{aligned}
$$

where

$$
\begin{gathered}
\omega_{\left(\lambda_{n}\right)}^{\mathcal{Z}}\left(\rho_{n}, \varepsilon\right)=\inf \left\{\mathcal{G} \geq 0:\left|\rho_{n}\left(\iota, \sigma_{1}, \ldots, \sigma_{n}, \ldots, v\right)-\rho\left(\iota+h, \sigma_{1}, \ldots, \sigma_{n}, \ldots, v\right)\right| \leq \mathcal{G}\right. \\
\text { for a.e. } \left.\iota \in \bar{B}_{\mathcal{Z}},\|h\| \leq \varepsilon,\left|\sigma_{i}\right| \leq \lambda_{i},|v|<D\right\}
\end{gathered}
$$

and

$$
\begin{gathered}
\omega_{\left(\lambda_{n}\right)}^{\mathcal{Z}}\left(\eta_{n}, \varepsilon\right)=\inf \left\{C \geq 0:\left|\eta_{n}\left(\iota, \kappa,\left(\sigma_{j}(\kappa)\right)_{j=1}^{\infty}\right)-\eta_{n}\left(\iota+h, \kappa,\left(\sigma_{j}(\kappa)\right)_{j=1}^{\infty}\right)\right| \leq C\right. \\
\text { for a.e. } \left.\iota, \kappa \in \bar{B}_{\mathcal{Z}} \cap \mathfrak{G},\|h\| \leq \varepsilon,\left|\sigma_{j}\right| \leq \lambda_{j}\right\} .
\end{gathered}
$$

Since $\sigma_{n}$ was an arbitrary element of $\Delta_{n}$ for all $n \in \mathbb{N}$ in the above inequality, we subsume that

$$
\begin{aligned}
\omega^{\mathcal{Z}}\left(H_{n}\left(\prod_{i=1}^{\infty} \Delta_{i}\right), \varepsilon\right) & \leq \omega_{\left(\lambda_{n}\right)}^{\mathcal{Z}}\left(\rho_{n}, \varepsilon\right)+\chi\left(\sup _{i \geq 1} \omega^{\mathcal{Z}}\left(\sigma_{i}, \varepsilon\right)\right) \\
& +\omega_{\left(\lambda_{n}\right)}^{\mathcal{Z}}\left(\eta_{n}, \varepsilon\right)+2 e s s \sup _{\iota \in \mathfrak{G}} \int_{\mathfrak{G} \backslash \bar{B}_{\mathcal{Z}}}\left|\eta_{n}\left(\iota, \kappa,\left(\sigma_{j}(\kappa)\right)_{j=1}^{\infty}\right)\right| d \kappa .
\end{aligned}
$$

Having Carathéodory provisions for $\rho_{n}$ and $\eta_{n}$ on $\bar{B}_{\mathcal{Z}} \times \prod_{i=1}^{\omega} \bar{B}_{\lambda_{i}} \times[-D, D]$ and $\bar{B}_{\mathcal{Z}} \times \bar{B}_{\mathcal{Z}} \times$ $\prod_{i=1}^{\infty} \bar{B}_{\lambda_{i}}$ respectively, one has $\omega_{\left(\lambda_{n}\right)}^{\mathcal{Z}}\left(\rho_{n}, \varepsilon\right) \rightarrow 0$ and $\omega_{\left(\lambda_{n}\right)}^{\mathcal{Z}}\left(\eta_{n}, \varepsilon\right) \rightarrow 0$ as $\varepsilon \rightarrow 0$. Thus, we procure that

$$
\omega_{0}^{\mathcal{Z}}\left(H_{n}\left(\prod_{i=1}^{\infty} \Delta_{i}\right)\right) \leq \chi\left(\sup _{i \geq 1} \omega_{0}^{\mathcal{Z}}\left(\Delta_{i}\right)\right)+2 \text { ess } \sup _{\iota \in \mathfrak{G}} \int_{\mathfrak{G}_{\mathfrak{B}} \backslash \bar{B}_{\mathcal{Z}}}\left|\eta_{n}\left(\iota, \kappa,\left(\sigma_{j}(\kappa)\right)_{j=1}^{\infty}\right)\right| d \kappa .
$$

If $\mathcal{Z} \rightarrow \infty$, handling supposition $\left(\mathcal{P}_{3}\right)$ we attain that

$$
\omega_{0}\left(H_{n}\left(\prod_{i=1}^{\infty} \Delta_{i}\right)\right) \leq \chi\left(\sup _{i \geq 1} \omega_{0}\left(\Delta_{i}\right)\right) .
$$

On the other hand, for all $\sigma_{i}, \varsigma_{i} \in \Delta_{i}$, we have

$$
\begin{aligned}
& \underset{\|\iota\|>\mathcal{Z}}{\operatorname{ess} \sup }\left|H_{n}\left(\left(\sigma_{j}\right)_{j=1}^{\infty}\right)(\iota)-H_{n}\left(\left(\varsigma_{j}\right)_{j=1}^{\infty}\right)(\iota)\right| \leq \underset{\|\iota\|>\mathcal{Z}}{\operatorname{ess} \sup }\left(\chi\left(\sup _{i \geq 1}\left|\sigma_{i}(\iota)-\varsigma_{i}(\iota)\right|\right)\right) \\
& +\underset{\|\iota\|>\mathcal{Z}}{+e s s \sup }\left|\int_{\mathfrak{G}}\left(\eta_{n}\left(\iota, \kappa,\left(\sigma_{j}(\kappa)\right)_{j=1}^{\infty}\right)-\eta_{n}\left(\iota, \kappa,\left(\varsigma_{j}(\kappa)\right)_{j=1}^{\infty}\right)\right) d \kappa\right| .
\end{aligned}
$$


Accordingly, we attain that

$$
\begin{aligned}
& d_{\mathcal{Z}}\left(H_{n}\left(\prod_{i=1}^{\infty} \Delta_{i}\right)\right) \leq \chi\left(\sup _{i \geq 1} d_{\mathcal{Z}}\left(\Delta_{i}\right)\right) \\
& +\underset{\|\iota\|>\mathcal{Z}}{+e s s \sup }\left|\int_{\mathfrak{G}}\left(\eta_{n}\left(\iota, \kappa,\left(\sigma_{j}(\kappa)\right)_{j=1}^{\infty}\right)-\eta_{n}\left(\iota, \kappa,\left(\varsigma_{j}(\kappa)\right)_{j=1}^{\infty}\right)\right) d \kappa\right| .
\end{aligned}
$$

If $\mathcal{Z} \rightarrow \infty$ in (3.18), exploiting $\left(\mathcal{P}_{3}\right)$ we have

$$
d\left(H_{n}\left(\prod_{i=1}^{\infty} \Delta_{i}\right)\right) \leq \chi\left(\sup _{i \geq 1} d\left(\Delta_{i}\right)\right)
$$

Subsequently, constituting (3.17) and (3.19) we acquire that

$$
d\left(H_{n}\left(\prod_{i=1}^{\infty} \Delta_{i}\right)\right)+H_{0}\left(H_{n}\left(\prod_{i=1}^{\infty} \Delta_{i}\right)\right) \leq \chi\left(\sup _{i \geq 1} H_{0}\left(\Delta_{i}\right)\right)+\chi\left(\sup _{i \geq 1} d\left(\Delta_{i}\right)\right) .
$$

Since $\chi$ is a concave function, from (3.20) we acquire that

$$
\begin{aligned}
\frac{1}{2}\left(\omega_{0}\left(H_{n}\left(\prod_{i=1}^{\infty} \Delta_{i}\right)\right)+\operatorname{diam}\left(H_{n}\left(\prod_{i=1}^{\infty} \Delta_{i}\right)\right)\right) & \leq \frac{1}{2}\left[\chi\left(\sup _{i \geq 1} \omega_{0}\left(\Delta_{i}\right)\right)+\chi\left(\sup _{i \geq 1} \operatorname{diam}\left(\Delta_{i}\right)\right)\right] \\
& \leq \chi\left(\frac{1}{2}\left(\sup _{i \geq 1} \omega_{0}\left(\Delta_{i}\right)\right)+\frac{1}{2} \limsup _{i} \operatorname{diam}\left(\Delta_{i}\right)\right),
\end{aligned}
$$

and we get

$$
\frac{1}{2} \mathfrak{M}_{n}\left(H_{n}\left(\prod_{i=1}^{\infty} \Delta_{i}\right)\right) \leq \chi\left(\frac{1}{2} \mathfrak{M}_{n}\left(\Delta_{i}\right)\right) .
$$

Taking $\mathfrak{M}_{n}^{\prime}=\frac{1}{2} \mathfrak{M}_{n}$, we find that

$$
\mathfrak{M}_{n}^{\prime}\left(H_{n}\left(\prod_{i=1}^{\infty} \Delta_{i}\right)\right) \leq \chi\left(\mathfrak{M}_{n}^{\prime}\left(\Delta_{i}\right)\right)
$$

Now, from Corollary 2.2 and taking $\zeta_{i}=\chi$ for all $i \in \mathbb{N}$, for a $\left(\sigma_{i}=\sigma_{i}(\iota)\right)_{i=1}^{\infty} \in$ $\left(L^{\infty}(\mathfrak{G})\right)^{\omega}$ one has

$$
\sigma_{n}(\iota)=\rho_{n}\left(\iota, \sigma_{1}(\iota) \ldots, \sigma_{n}(\iota), \ldots, \int_{\mathfrak{G}} \eta_{n}\left(\iota, \kappa,\left(\sigma_{j}(\kappa)\right)_{j=1}^{\infty}\right) d \kappa\right) .
$$

Example Let:

$$
\begin{aligned}
\sigma_{n}(\iota) & =\frac{\|\iota\|_{\infty}^{2}}{2+\|\iota\|_{\infty}^{4}}+\tanh \left|\sigma_{n}(\iota)\right| \\
& +\frac{1}{e^{\|\iota\|_{\infty}}} \arctan \left(\int_{\mathfrak{G}} \frac{\sin \left(\|\kappa\|_{\infty}^{3}\right) \cdot \cos \left(\left(\sigma_{n}(\iota)\right)_{n=1}^{\infty}\right)+\cos ^{3}\left(\|\iota\|_{\infty}\right) \cdot \sin \left(\left(\sigma_{n}(\iota)\right)_{n=1}^{\infty}\right)}{3} d \kappa\right),
\end{aligned}
$$

where $i, n \in \mathbb{N}$. Eq. (3.21) is a special case of Eq. (1.1) with

$$
\begin{gathered}
\rho_{n}\left(\iota, \sigma_{1}, \ldots, \sigma_{n}, \ldots, z\right)=\frac{\|\iota\|_{\infty}^{2}}{2+\|\iota\|_{\infty}^{4}}+\tanh \left|\sigma_{n}(\iota)\right|+\frac{1}{e^{\|\iota \iota\|_{\infty}}} \arctan z \\
\eta_{n}\left(\iota, \kappa, \sigma_{1}, \sigma_{2}, \ldots\right)=\frac{\sin \left(\|\kappa\|_{\infty}^{3}\right) \cdot \cos \left(\left(\sigma_{n}(\iota)\right)_{n=1}^{\infty}\right)+\cos ^{3}\left(\|\iota\|_{\infty}\right) \cdot \sin \left(\left(\sigma_{n}(\iota)\right)_{n=1}^{\infty}\right)}{3}
\end{gathered}
$$

and $\mathfrak{G} \subseteq \mathbb{R}^{\omega}$ is bounded.

Evidently $\left(\mathcal{P}_{1}\right)$ holds and $\chi(t)=\arctan (t)$ is nondecreasing, concave and upper semicontinuous such that $\chi(t)<t$ for a.e. $t>0$. Let $\iota \in \mathbb{R}$ and $\sigma_{i} \geq \varsigma_{i}$. Thus 


$$
\begin{aligned}
& \left|\rho_{n}\left(\iota, \sigma_{1}, \ldots, \sigma_{n}, \ldots, \varrho\right)-\rho_{n}\left(\iota, \varsigma_{1}, \ldots, \varsigma_{n}, \ldots, q\right)\right| \\
& \leq|\tanh | \sigma_{n}(\iota)|-\tanh | \varsigma_{n}(\iota)|| \\
& +\frac{1}{e^{\|l \iota\|_{\infty}}}|\arctan \varrho-\arctan q| \\
& \leq \tanh \left|\sigma_{n}(\iota)-\varsigma_{n}(\iota)\right|+\frac{1}{e^{\| l \iota \mid \infty}}|\varrho-q| \\
& \leq \tanh \sup _{n \geq 1}\left|\sigma_{n}(\iota)-\varsigma_{n}(\iota)\right|+|\varrho-q| \\
& =\chi\left(\sup _{n \geq 1}\left|\sigma_{n}(\iota)-\varsigma_{n}(\iota)\right|\right)+|\varrho-q| .
\end{aligned}
$$

As well as,

$$
\left.E_{n}:=\text { ess } \sup _{\iota \in \mathfrak{G}}\left|\rho_{n}(\iota, 0,0, \ldots)\right|: n \in \mathbb{N}\right\}=\text { ess } \sup _{\iota \in \mathfrak{G}}\left\{\frac{\iota^{2}}{2+\iota^{4}}\right\} \leq 0.36
$$

Also, $\rho$ satisfies the Carathéodory supposition and $\rho_{n}(., 0,0) \in L^{\infty}\left(\mathbb{R}^{\omega}\right)$. Thus, condition (ii) holds. Moreover, $\eta_{n}$ satisfies the Carathéodory conditions and since

$$
\begin{aligned}
& \text { ess } \sup _{\iota \in \mathfrak{G}}\left|\int_{\mathbb{R}} \eta_{n}\left(\iota, \kappa,\left(\sigma_{j}(\kappa)\right)_{j=1}^{\infty}\right) d \kappa\right| \\
& =e s s \sup _{\iota \in \mathbb{R}} \mid \frac{1}{e^{\iota}}\left(\int_{\mathbb{R}} \frac{\sin \left(\kappa^{3}\right) \cdot \cos \left(\sigma_{n}(\iota)\right)+\cos ^{3}(\iota) \cdot \sin \left(\sum_{i=1}^{\infty} \frac{1}{\left|\sigma_{i}(\iota)\right|^{2}+1}\right)}{\left(2+\sin \left(\sum_{i=1}^{\infty} \frac{1}{\left|\sigma_{i}(\iota)\right|^{2}+1}\right)\right.} d \kappa \mid\right. \\
& \leq \frac{2}{e},
\end{aligned}
$$

so, we take $D=\frac{2}{e}$. Moreover,

$$
\begin{aligned}
\lim _{\mathcal{Z} \longrightarrow \infty} \operatorname{ess} \sup & \mid \frac{1}{\|\iota\|>\mathcal{Z}}\left(\int_{\mathbb{R}} \frac{\sin \left(\kappa^{3}\right) \cdot \cos \left(\sigma_{n}(\iota)\right)+\cos ^{3}(\iota) \cdot \sin \left(\sum_{i=1}^{\infty} \frac{1}{\left|\sigma_{i}(\iota)\right|^{2}+1}\right)}{\left(2+\sin \left(\sum_{i=1}^{\infty} \frac{1}{\left|\sigma_{i}(\iota)\right|^{2}+1}\right)\right.}\right. \\
& -\frac{\sin \left(\kappa^{3}\right) \cdot \cos \left(\varsigma_{n}\right)+\cos ^{3}(\iota) \cdot \sin \left(\sum_{i=1}^{\infty} \frac{1}{\left|\varsigma_{i}\right|^{2}+1}\right)}{\left(2+\sin \left(\sum_{i=1}^{\infty} \frac{1}{\left|\varsigma_{i}\right|^{2}+1}\right)\right.} d \kappa \mid=0
\end{aligned}
$$

uniformly with respect to $\sigma_{j}, \varsigma_{j} \in L^{\infty}(\mathbb{R})$ and for all $\lambda>0$ with $\max \left\{\left\|\sigma_{j}\right\|_{\infty},\left\|\varsigma_{j}\right\|_{\infty}\right\} \leq \lambda$. Also,

$$
\lim _{\mathcal{Z} \longrightarrow \infty} e s s \sup _{\iota \in \mathbb{R}}\left|\int_{\mathbb{R} \backslash \bar{B}_{\mathcal{Z}}} \frac{1}{e^{\iota}} \frac{\sin \left(\kappa^{3}\right) \cdot \cos \left(\sigma_{n}(\iota)\right)+\cos ^{3}(\iota) \cdot \sin \left(\sum_{i=1}^{\infty} \frac{1}{\left|\sigma_{i}(\iota)\right|^{2}+1}\right)}{\left(2+\sin \left(\sum_{i=1}^{\infty} \frac{1}{\left|\sigma_{i}(\iota)\right|^{2}+1}\right)\right.} d \kappa\right|=0 .
$$

It is easy to check that the sequence $\left(\lambda_{n}\right)=(3,4,5, \ldots)$ satisfies the inequality in condition $\left(\mathcal{P}_{4}\right)$, i.e.,

$$
\chi\left(\sup _{n \geq 1} \lambda_{n}\right)+E_{n}+D=0.99505475368+1+\frac{2}{e} \leq \lambda_{n},
$$

for all $n$. Therefore, all the conditions of Theorem 3.4 hold. Hence, the integral equation (3.21) possesses at least a solution.

\section{CONCLUSION}

The existence of a solution for the integral equation system (1.1) in the space $L^{\infty}(G)$, where $G \subseteq \mathbb{R}^{\omega}$, was investigated in this study. Arab et al. [5] investigated the problem of finding solutions for an infinite system of two-variable integral equations in the space $\left(B C\left(\mathbb{R}_{+} \times \mathbb{R}_{+}\right)\right)^{\omega}$. The benefit of our approach is that any function in the space $L^{\infty}(G)$ need not to be continuous. 


\section{REFERENCES}

[1] Agarwal, R. P.; Meehan, M.; O'Regani, D. Fixed point theory and applications, Cambridge University Press, 2004.

[2] Aghajani, A.; Allahyari, R.; Mursaleen, M. A generalization of Darbo's theorem with application to the solvability of systems of integral equations. J. Comput. Appl. Math. 260 (2014) 67-77

[3] Allahyari, R. The behaviour of measure of noncompactness in $L^{\infty}\left(R^{n}\right)$ with application to the solvability of functional integral equations. Rev. R. Acad. Cienc. Exactas Fis. Nat. Ser. A Math. RACSAM 112 (2018), no. 2, 561-573.

[4] Allahyari, R.; Arab, R.; Haghighi, A. S. Existence of solutions of infinite systems of integral equations in the Fréchet spaces. Int. J. Nonlinear Anal. Appl. 7 (2016), no. 2, 205-216

[5] Arab, R.; Allahyari, R.; Haghighi, A. S. Existence of solutions of infinite systems of integral equations in two variables via measure of noncompactness. Appl. Math. Comput. 246 (2014) 283-291.

[6] Banaei, Sh. Solvability of a system of integral equations of Volterra type in the Frechet space $L_{l o c}^{p}\left(R_{+}\right)$via measure of noncompactness. Filomat 32 (2018), no. 15, 5255-5263.

[7] Banaei, Sh. An extension of Darbo's theorem and its application to existence of solution for a system of integral equations. Cogent Math Stat. 6 (2019), no. 1, doi: 10.1080/25742558.2019.1614319.

[8] Banaś, J.; Geobel, K. Measure of Noncompactness in Banach Spaces, Lecture Notes in Math. 60, Dekker, New York, 1980.

[9] Darbo, G. Punti uniti in trasformazioni a codominio non compatto. Rend. Semin. Mat. Univ. Padova 24 (1955), 84-92

[10] Das, A.; Hazarika, B.; Arab, R.; Mursaleen, M. Solvability of the infinite system of integral equations in two variables in the sequence spaces $c_{0}$ and $\ell_{1}$. Jour. Comput. Appl. Math. 326 (2017), 183-192.

[11] Das, A.; Hazarika, B.; Mursaleen, M. Application of measure of noncompactness for solvability of the infinite system of integral equations in two variables in $\ell_{p}(1<p<\infty)$. Rev. R. Acad. Cienc. Exactas Fis. Nat. Ser. A Math. RACSAM 113 (2019), no. 1, 31-40

[12] Das, A.; Rabbani, M.; Hazarika, B.; Arab, R. Solvability of infinite system of nonlinear singular integral equations in the $C(I \times I, c)$ space and modified semi-analytic method to find a closed-form of solution. Int. J. Nonlinear Anal. Appl. 10 (2019), no. 1, 63-76

[13] Dugundji, J. Topology (Allyn and Bacon Series in Advanced Mathematics), Allyn and Bacon, Inc, 1966.

[14] Hazarika, B.; Arab, R.; Mursaleen, M. Applications of measure of noncompactness and operator type contraction for existence of solution of functional integral equations. Complex Anal. Oper. Theory 13 (2019), 3837-3851

[15] Hazarika, B.; Srivastava, H. M.; Arab, R.; Rabbani, M. Existence of solution for an infinite system of nonlinear integral equations via measure of noncompactness and homotopy perturbation method to solve it. $J$. Comput. Appl. Math. 343 (2018), 341-352.

[16] Jarchow, H. Locally Convex Spaces, Teubner, Stuttgart, 1981.

[17] Kuratowski, C. Sur les espaces complets. Fund. Math. 15 (1930), no. 1, 301-309

[18] Maleknejad, K.; Torabi, P.; Mollapourasl, R. Fixed point method for solving nonlinear quadratic Volterra integral equations. Comput. Math. Appl. 62 (2011)., no. 6, 2555-2566

[19] Mursaleen, M.; Rizvi, S. M. H. Solvability of infinite system of second order differential equations in $c_{0}$ and $\ell_{1}$ by Meir-Keeler condensing operator. Proc. Amer. Math. Soc. 144 (2016), no. 10, 4279-4289.

[20] Olszowy, L. Solvability of some functional integral equation. Dynam. Systems Appl. 18 (2009), no. 3, 667-676.

[21] Olszowy, L. Solvability of infinite systems of singular integral equations in Fréchet space of continuous functions. Comput. Math. Appl. 59 (2010), 2794-2801.

${ }^{1}$ Department of Mathematics

BONAB BRANCH, IsLAMIC AZAD UNIVERSITY, BONAB, IRAN

Email address: math.sh.banaei@gmail.com

2 Department of Mathematics

GILAN-E-GHARb Branch, Islamic AZAD University, GILAN-E- GHARb, Iran

Email address: zam.dalahoo@gmail.com

${ }^{3}$ China Medical University Hospital, China Medical University

DepartMent of Medical Research

TAICHUNG, TAIWAN

${ }^{4}$ Department of Mathematics

Aligarh Muslim University, Aligarh-202 002, INDiA

Email address: mursaleenmegmail .com 